(C) 1981. The Genetical Society of Great Britain

\title{
THE EFFECT OF PARENTAL AGE ON STERNO- PLEURAL CHAETA NUMBER IN DROSOPHILA MELANOGASTER
}

\author{
P. D. S. CALIGARI* and D. F. A. BABAN \\ Department of Genetics, University of Birmingham, P.O. Box 363, Birmingham, \\ B15 2TT, U.K.
}

Received 15.i.81.

\section{SUMMARY}

\begin{abstract}
An experiment is presented which produced variation in sternopleural chaetae number related to the age of the parent flies thus giving results in accordance with those indicated by the changes in heritability described by Beardmore, Lints and Al-Baldawi (1975). It is shown, however, that this does not result from a direct effect of parental age on chaeta number but rather reflects an indirect effect of parental age through the number of progeny produced, that is an effect of density of culture in which the larvae develop.
\end{abstract}

\section{INTRODUCTION}

IT has been reported (Beardmore, Lints and Al-Baldawi, 1975; Beardmore, 1976; Lints, 1978) that the heritability of sternopleural chaeta number in Drosophila melanogaster is influenced by the age of the parent flies. Their results were surprising in that they showed the heritability to be higher among flies produced from parents which were 14 days or older than from parents with a mean age of 3 days. In other words heritability increased with increasing parental age, which as pointed out by Lints (l.c.), would seem to run contrary to expectations based on most of the accepted theories of ageing. It was suggested that this was in part due to a reduction in the effects of random environmental variation on the progeny but mainly to direct genetic effects such as reduced recombination, changes in meiotic drive, alteration in the quantity of genetic material in the gametes or inherited changes in additive gene action. It was also claimed later (Shami, 1977) that such age dependent effects were shown mainly by parents with central rather than extreme phenotypes for a trait from which it was argued that the effect was likely to be associated with heterozygosity.

The existence of such direct genetic effects attributable to parental age is clearly of potential interest not only in terms of the ageing process itself but also the consequences for evolution as well as the efficacy of artificial selection in breeding programmes. However, since the experiments were carried out on samples taken directly from a laboratory population and the subsequent analyses carried out on estimates of the heritability, no detailed test of the underlying mechanisms was possible. The aim of the present investigation was to attempt to reproduce the ageing effects described by the earlier workers but using inbred lines and in this way study their underlying causation. The advantages of using inbred lines are

* Present Address: Scottish Crop Research Institute, Pentlandfield, Roslin, Midlothian EH25 9RF. 
that they can be maintained unchanged for further detailed study, experiments can readily be replicated and crosses of known genetic constitution can be made such that comparisions between different levels of homozygosity/heterozygosity can be carried out.

\section{EXPERIMENTS}

Several preliminary experiments were carried out to investigate the progeny of different aged parents using inbred lines and various generations derived from crosses amongst them. The inbred lines employed were derived from a laboratory population, "Texas" (see Caligari and Mather, 1980). These early experiments will not be described here but they showed that a difference in sternopleural chaetae number could be detected between the progeny of young and old parents, in accordance with the previous workers' findings. They also showed, however, that the repeatability of the effect for any genotype on different occasions was low. There was no discernible difference between the behaviour of homozygous as opposed to heterozygous parents and no significant effect traceable to the age of the grandparents. These preliminary experiments also allowed the choice of two inbred lines to be made which showed, on average, the most dissimilar effects of parental ageing and they led to the observation that parental flies of different ages tended to produce different numbers of progeny.

On the basis of these preliminary findings an experiment was set up using the two lines selected and the reciprocal first crosses between them (Texas 1 , Texas 28 , Texas $1 \times$ Texas 28 and Texas $28 \times$ Texas 1 ). The experimental conditions were basically the same as those described by Beardmore et al. (l.c.) with the ageing carried out at $18^{\circ} \mathrm{C}$ and all the progeny raised at $25^{\circ} \mathrm{C}$. Three ages of parent were investigated: less than 3 days (Young), between 8 and 10 days (Middle age) and between 15 and 17 days old (Old). Because of the observed differences in the production of progeny in relation to parental age the experiment was set up in two halves. In the first half flies of each genotype were mated and at the appropriate age, allowed to lay for 48 hours in tubes with standard oatmealmolasses-agar medium, the surface of which had been previously seeded with yeast. For young and middle aged flies the matings were of 7 males with 7 females but for old, because (particularly where Texas 28 mothers were involved) it was found that very few progeny resulted, this was increased to 10 couples. In the second half, flies at each of the three ages, ( 3,10 or 17 days old) were allowed to lay eggs for 4 hours on charcoal blackened starch sticks. For every combination of age and genotype 70 of the eggs so produced were counted and placed in tubes containing the same medium as the other half of the experiment. In all other respects the two halves of the experiment were treated in an identical manner as a single randomised experiment. In each half of the experiment for every combination of age and genotype, 4 replicates were produced on each of two days thus giving 96 cultures in all. From each of these cultures 10 females were taken at random from the progeny produced and scored for sternopleural chaeta number. The whole experiment was repeated on 4 separate occasions. Thus, taking the mean of the 10 flies as the basic datum, for each half of the experiment there are 4 (genotypes) $\times 3$ (ages) $\times 2$ $($ days $) \times 4$ (occasions $) \times 4$ (replicates $)=384$ observations. 
The analyses of variance of the two halves of the experiment are shown separately in table 1 , (i) referring to the data from cultures where the parents were allowed to lay freely for 48 hours while (ii) refers to the cultures produced by the 70 counted eggs. The error variance of each half of the experiment has 288 degrees of freedom (d.f.). The estimates from the two halves showed no significant difference, however, and so were pooled to give a joint estimate of error based on $576 \mathrm{~d}$.f. This pooled error variance has been used to test all the items in both analyses.

TABLE 1

The analysis of sternopleural chaeta number among offspring produced by Young, Middle and Old parents (i) Parents allowed to lay for 48 hours (ii) 70 eggs placed in each tube

(i)

$\quad$ Item
Ages $(A)$
Genotypes $(G)$
Days $(D)$
Occasions $(\mathrm{O})$
$A \times G$
$A \times D$
$A \times O$
$G \times D$
$G \times O$
$D \times O$
$A \times G \times D$
$A \times D \times O$
$A \times G \times O$
$G \times D \times O$
$A \times G \times D \times O$
$R e p l i c a t e$ error

d.f.
2
3
1
3
6
2
6
3
9
3
6
6
18
9
18
576

M.S.
21.60
646.61
2.39
0.68
1.02
0.58
1.72
0.57
0.11
1.49
0.08
0.42
0.67
0.42
0.20

$\begin{array}{cr}\text { P } & \text { M.S. } \\ \ll 0.001 & 0.06 \\ \ll 0.001 & 696.86 \\ \simeq 0.01 & 0.80 \\ \text { N.S. } & 0.33 \\ 0.02-0.01 & 0.49 \\ \text { N.S. } & 0.62 \\ <0.001 & 0.87 \\ \text { N.S. } & 0.41 \\ \text { N.S. } & 0.26 \\ 0.01-0.001 & 0.25 \\ \text { N.S. } & 0.15 \\ \text { N.S. } & 0.22 \\ 0.02-0.01 & 0.31 \\ \text { N.S. } & 0.21 \\ \text { N.S. } & 0.24 \\ \text { M.S. }=0.3667\end{array}$

(ii)

P
n.s.
$\ll 0 \cdot 001$
N.S.
N.S.
N.S.
N.S.
$0 \cdot 05-0 \cdot 02$
N.S.
N.S.
N.S.
N.S.
N.S.
N.S.
N.S.
N.S.

Considering first the mean squares (M.S.) in (i), it can be seen that the overall difference in chaeta number between the genotypes $(G)$ accounts for the major portion of the variation. The next largest M.S. is, however, the effect of parental age $(A)$ and this varies significantly with genotype $(A \times G)$ thus agreeing with our preliminary findings and those of the earlier workers. The other four significant items, days $(D)$, ages $\times$ occasions $(A \times O)$, days $\times$ occasions $(D \times O)$ and ages $\times$ genotypes $\times$ occasions $(A \times G \times O)$ all reflect the effects of uncontrolled environmental variation.

When attention is turned to (ii) where the density was fixed by using counted eggs it can be seen that the difference between genotypes is again highly significant. However, neither the effect of ageing nor its interaction with genotypes approach significance. The only other item which is significant is the interaction of ages with occasions $(A \times O)(P=0.03)$. Since the probability is close to 5 per cent and neither main effect even approaches significance it is doubtful considering the number of tests carried out, whether this item's significance should be regarded as other than attributable to sampling error. Thus, when the density of the cultures is controlled the effect of parental age on sternopleural chaeta number not only decreases but effectively vanishes. 


\section{EgG PRODUCTION AND HATCHABILITY}

By fixing the number of eggs in the cultures, parental age effects are no longer detectable and it would seem reasonable to conclude that they are related to the density of larvae in the culture. It would be expected therefore that a significant relationship would exist within a genotype between the sternopleural chaeta number of offspring in (i) and the density of the culture. This proposition can be investigated since when the 70 eggs were transferred to produce each of the cultures in (ii) the remaining eggs on the starch stick were also recorded; thus adding the two together gives a measure of egg production. By dividing this measure by the number of females that were laying and the number of hours for which they were laying, an expected egg production per female per hour can be calculated for each culture. Furthermore, the hatchability of the eggs of each culture was also assessed by transferring 25 of them onto charcoal-blackened agar in a petri-dish and recording the numbers that hatched after 36 hours. Hence the expected egg production can be corrected for hatchability and an estimate related to the expected number of larvae per culture obtained allowing for the differing numbers of parents used. This should provide at least a reasonably close approximation to the variation in density between the parents of different ages.

Since interest centres on the relationship between chaeta number and expected number of larvae within a genotype, both these characters can be averaged over days, replicates and occasions to give the means in table 2 . The linear regression analysis of chaeta number on expected number of larvae can then be carried out for each genotype separately and the results combined to show the variation in chaeta number accounted for by the relationship with expected number of larvae, the heterogeneity of this

TABLE 2

Mean sternopleural chaeta number (above) and expected number of larvae (below)

\begin{tabular}{|c|c|c|c|}
\hline \multirow{2}{*}{$\underset{\substack{q \times \delta^{2} \\
\text { Genotype }}}{ }$} & \multicolumn{3}{|c|}{ Parental age } \\
\hline & Young & Middle & Old \\
\hline Tex $1 \times \operatorname{Tex} 1$ & $\begin{array}{r}16.90 \\
1.99\end{array}$ & $\begin{array}{r}16.45 \\
2 \cdot 74\end{array}$ & $\begin{array}{r}16.27 \\
3.45\end{array}$ \\
\hline Tex $1 \times$ Tex 28 & $\begin{array}{r}18.50 \\
1.99\end{array}$ & $\begin{array}{r}17 \cdot 63 \\
3 \cdot 16\end{array}$ & $\begin{array}{r}17 \cdot 38 \\
3.47\end{array}$ \\
\hline Tex $28 \times \operatorname{Tex} 1$ & $\begin{array}{r}18.69 \\
1.10\end{array}$ & $\begin{array}{r}18 \cdot 17 \\
1 \cdot 33\end{array}$ & $\begin{array}{r}18.34 \\
1.05\end{array}$ \\
\hline Tex $28 \times \operatorname{Tex} 28$ & $\begin{array}{r}23 \cdot 12 \\
0.99\end{array}$ & $\begin{array}{r}22.69 \\
1.64\end{array}$ & $\begin{array}{r}22.22 \\
1.46\end{array}$ \\
\hline
\end{tabular}

For each genotype and age the upper entry is the mean sternopleural chaeta number when the parental flies were allowed to lay freely for 48 hours. The lower entry is the expected number of larvae, obtained from the egg production per female per hour corrected for hatchability and varying parental numbers. Both entries are means obtained by averaging over the results for 2 days, 4 replicates and 4 occasions. 
TABLE 3

Joint regression analysis of sternopleural chaeta number on expected number of larvae

\begin{tabular}{lcrc}
\multicolumn{1}{c}{ Item } & d.f. & M.S. & P \\
Joint regression & 1 & $37 \cdot 97$ & $0 \cdot 01-0 \cdot 001$ \\
Heterogeneity between genotypes & 3 & $2 \cdot 14$ & N.S. \\
Remainder & 4 & 1.22 &
\end{tabular}

relationship over genotypes and the variation left unaccounted. The results of such an analysis are shown in Table 3, but it should be noted that the analysis was carried out on sums rather than means to make it comparable with the analyses in table 1 since it actually provides a repartitioning of the $A$ and $A \times G$ items of (i). The only estimate of error variation that is available in the experiment is the replicate error variance of table 1 but unfortunately this is not appropriate to the present analysis. The reason for this is that the regression analysis of variance assumes no error variation in the independent variate, which in the present case is manifestly not so. The replicate error variance would, therefore, be an underestimate in the present analysis.

As can be seen from the table the heterogeneity M.S. is not significantly greater than the remainder M.S. The joint regression item is significant and its sum of squares (S.S.) accounts for some 77 per cent of the total S.S. Therefore, in the present experiment all the variation in chaeta number produced by parents of different ages can be accounted for in terms of variation in the expected number of larvae which has been taken as a measure of density. Table 2 shows that while chaeta number is determined by the individual's genotype with dominance tending towards low numbers, expected number of larvae is largely maternally determined as might be anticipated since it is known that both egg production and hatchability, from which it was estimated, are determined in this way. The regression analysis makes clear that no matter how the differences in density are achieved they produce the same effect on chaeta number, as would indeed follow from the results of Caligari and Mather (1975).

\section{Conclusions}

The experiment reported here produced detectable variation in the sternopleural chaeta number of offspring related to the age of the parental files which would lead to just the sort of changes in heritability reported by Beardmore et al. (l.c.). When the number of eggs per culture was controlled, however, this variation disappeared. Moreover, the variation in chaeta number over parental age in cultures where the number of eggs was not controlled could entirely be accounted for by the effect of density measured as expected number of larvae. Thus the present experiment provides no evidence of any direct genetic effects of parental age on the expression of sternopleural chaeta number among the genotypes studied. At the same time it makes clear that if it is desired to attempt the detection of such effects, the experiments must necessarily be designed in such a way as to eliminate the indirect effects of age due to differential fecundity. 
Acknowledgements.-We are grateful to Professor Sir Kenneth Mather for his advice and encouragement during the course of this work. Financial support to one of us (P.D.S.C.) from the Agricultural Research Council is acknowledged.

\section{REFERENCES}

BEARDMORE, J. A. 1976. Genetics and ageing. Eugen, Soc. Bul., 8, 39-42.

BEARDMORE, J. A., LINTS, F, A., AND AL-BALDAWI, A. L. F. 1975. Parental age and heritability of sternopleural chaeta number in Drosophila melanogaster. Heredity, 34, 71-82.

CALIGARI, P. D.S., AND MATHER, K. 1975. Genotype-environment interaction. III. Interactions in Drosophila melanogaster. Proc. Roy. Soc. Lond. B., 191, 387-411.

CAligARI, P. D. S., AND MATHER, K. 1980. Dominance, allele frequency and selection in a population of Drosophila melanogaster. Proc. Roy. Soc. Lond. B., 208, 163-187.

LINTS, F. A. 1978. Genetics and ageing. In Interdisciplinary Topics in Gerontology., 14, ed. H. P. von Hahn. Karger, Basel.

SHAMI, S. A. 1977. Stabilizing selection, heterozygosity and parental age effects in the guppy Poecilia reticulata (Peters) and Drosophila melanogaster. Ph.D. Thesis, Swansea. 